\title{
The Computer-assisted Implementation of Three-dimensional Animation
}

\author{
Ma Jingfeng, Dong Haibo \\ Weifang University of Science and Technology, Shouguang, Shandong, China
}

\author{
Keywords: Three-dimensional Animation, Scripting Language, Effect, Maya
}

\begin{abstract}
The thesis proposes that there exists a more concise method to control the properties of fluid through analyzing and studying Maya Fluid Effects, after taking advantage of maxscript scripting language program to control the motion trails of the objects in three-dimensional animation. Through practice, we get the tips of particle driven fluid, which makes practical value on three-dimensional animation and performs the effect software of three-dimensional animation conveniently as well as accurately. Three-dimensional animation effects technology makes continuous innovation in animated movies, whose significance provides theoretical and practical basis for the technology of animation industry in the future.
\end{abstract}

\section{Introduction}

For the past few years, with the advent of digital era, computer animation develops rapidly. A lot of computer animations appear on the internet, which have complex types, various contents and thousands of ways to express feelings, so as to increasingly become a popular multimedia form. As one of the common images in everyday life, computer animation image offers people a refreshing experience and attracts a great deal of attention and interest, so the correlation studies of computer animation has gradually increased a great deal.

Computer animation is composed of a variety of elements, including image, sound, text and so on, among which the most basic element is image. Computer animation contains a large number of images, which not only make up the main body of computer animation, but express rich contents as well as consist flexible forms. The producers also express different feelings through these images. Our understanding of images can be divided into three levels. The first level is the visual perception of image, such as color, texture, shape and other visual features; the second level is the semantic entity of the first level, like vehicles, buildings, foods and so on; the third level is a abstract conception based on comprehension, which expresses with concrete concept, such as pretty, generous and so on. Our emotional tendency for computer animation image just belongs to the third level. In recent years, people come to realize the importance of making subjective sentiment analysis of image. In this way, classification research of image emotion develops rapid as well.

On the basis of comprehensive comparison between the method and process of making animation, the thesis considers the prominent advantages possessed by scripting language in the process of making some complex images. Through deep analysis by using the scripting language in the three-dimensional animation software 3D MAX, we complete three-dimensional scene modeling, and solve one sort of issue based on physical animation implementation in threedimensional animation. With analysis and studies of maya fluid effect, we obtain a more concise method of controlling fluid features. Combine particle to work on fluid effect, which can not only make fluid effect construct the sense of reality, but possess the control of particle.

\section{The feature extraction of computer animation image}

The colour feature of computer animation image. The colour feature of image has great influence on the its emotion, which is the most direct factor for people to judge image emotional tendency. Different colours make people produce different emotional tendencies. For instance, red stands for ardency, joy and enthusiasm; green strands for peace and vitality; blue stands for tranquillity, wisdom and profundity, etc. Accurately extracting the colour feature parameters of image is the foundation of labelling image emotional feature. 
The received computer animation image is described in RGB colour space. RGB colour space refers to red, green and blue colour space model, which is usually used in image pickup system, monitor and other hardware display device. It doesn't signify colour with consistency measure, which is not in accordance with human perception of colour psychology. Therefore, we need to search another colour space model which is more suitable. HSV colour space model possesses visual consistency, and compared with RGB colour space system, it is more aligned with human visual system. Similarly, HSV colour space model also have three dimensions, which stand for three colours at the same time.

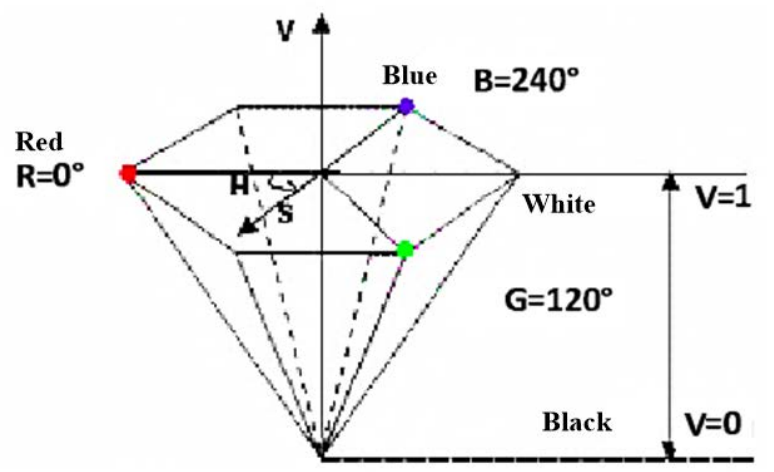

Fig.1 HSV color model

Use HSV color space model to describe the visual feature of computer animation image. Suppose R, G, B $\in[0,1, \ldots, 255]$, and the following is the conversion from RGB color space to HSV color space:

Define r=R255, g=G255, b =B255. Suppose m=max (r , g, b), n=min ( r, g, b)

$$
H=\left\{\begin{array}{l}
0, m=n \\
60(g-b) /(m-n)+120, g=m \\
60(r-g) /(m-n)+240, b=m \\
60(g-b) /(m-n), r=m \& g \geq b \\
60(g-b) /(m-n), r=m \& g<b
\end{array}\right.
$$

The image selected from computer animation is true color image. Each image contains plenty of colors. When counting colors with color histogram, there are usually many dimensionalities, which is not convenient to describe the color of image. According to quantitative series, the new color value formed by three color components is $\left(\mathrm{Q}_{\mathrm{s}}\right.$ and $\mathrm{Q}_{\mathrm{V}}$ are the quantitative series of saturation $\mathrm{S}$ and brightness $\mathrm{V}$ respectively.)

$$
\mathrm{L}=\mathrm{HQ}_{\mathrm{s}} \mathrm{Q}_{\mathrm{V}}+\mathrm{SQ}_{\mathrm{V}}+\mathrm{V}
$$

The textural features of computer animation image. Image edge detection is a method of searching edge by judging the discrepancies between neighbors. The basic idea is to detect the edge points appearing in the image, then connect these edge points into a contour according to certain strategy, thus, form the edge image. In this thesis, for computer animation image, we adopt the idea of image edge detection to obtain the contour formed by edge points, which is called side boundary. Among these, Robert operator is a basic operator in edge detection, whose rationale is to searching edge points by using part difference operator, so as to form edge image.

To describe color image in certain color space, each image will produce several color channels. And on each color channel is quite a gray level image. We can calculate with Robert operator which can use gray level image and summate the Robert operators in each color channel, so as to form the space Robert operator of color image edge point detection. Take the following three color channels as an example, and the expression is: 


$$
\begin{aligned}
& G[i, j]=|f[i, j, 1]-f[i+1, j+1,1]|+|f[i, j, 2]-f[i+1, j+1,2]| \\
& +|f[i, j, 3]-f[i+1, j+1,3]|+|f[i+1, j, 1]-f[i, j+1,1]| \\
& +|f[i+1, j, 2]-f[i, j+1,2]|+|f[i+1, j, 2]-f[i, j+1,2]|
\end{aligned}
$$

In this expression, $G[i, j]$ signifies the gray level of point $(i, j)$ after counting; $f[i, j, 1]$ signifies its $R$ component value before counting; $f[i, j, 2]$ signifies its $G$ component value before counting; $f$ $[i, j, 3]$ signifies its B component value before counting. As shown in Fig.2, we need to do normalization processing of R, G, B color components of image, with the purpose of making their component values locate between zero and one, which is beneficial to the quantification of image side boundary. Then, through space Roert operator formula metioned above, we can calculate the Robert operator value of all pixels in computer animation image. Summate the Rebert operator values of all pixeds, and then divide pixel count of the whole image, so as to obtain the average value of Robert operator in computer animation image.

\begin{tabular}{|l|l|} 
Image input & The normalized \\
color space
\end{tabular}$\longrightarrow \begin{gathered}\text { Calculation of } \\
\text { Robert operator }\end{gathered} \longrightarrow \rightarrow \begin{gathered}\text { Robert operator sum and } \\
\text { average value }\end{gathered}$

Fig.2 flow chart of Robert operator mean value of image

The emotional feature of computer animation image.The emotional feature of image refers to common emotional tendencies produced by people for certain things. Different kinds of images are described by different emotional models. The research object of the thesis is computer animation image, so specific to its basic feature and applied environment, we confirm 16 emotional models, which are warm and sweet, tranquil, lively, active, funny, exaggerated, humorous, amusing, lonely, bald, depressing, mess, illusory, thrilling, horrible and intense. The accuracy of emotional model directly influences the map mechanism of visual and emotional features of images, and then, further influences the labels of emotional features in computer animation image.

Different image features usually make people produce different emotions, among which color and textural feature are main factors. When the emotion is warm and sweet, warm tone is dominant; when the emotion is depressing, cold tone has the upper hand, and the complexity of side boundary between them is low. When the emotion is lively or amusing, the colors of image are bright and various, and the complexity of the side boundary is moderate. When the emotion is exaggerated or mess, the color is bright as well, but the complexity of side boundary is obviously much higher, and the layout of image is kind of full, which makes it more visually striking. When the emotion is lonely, the tone is obvious dark, and there is no highlighted color tendency. When the emotion is illusory, the side boundary is not obvious, and there is no clear classification, while the integral emotion is stronger.

\section{Three-dimensional animation technology based on scripting language}

The composition of three-dimensional animation system.As shown in Fig.3, the composition of three-dimensional animation includes data acquisition system, modelling system, surface material editing system, animation system, the rendering system, multimedia information processing synthetic system, the host computer or workstation, input devices, scanners, cameras, digital instrument etc., output device film output equipment, video equipment. 


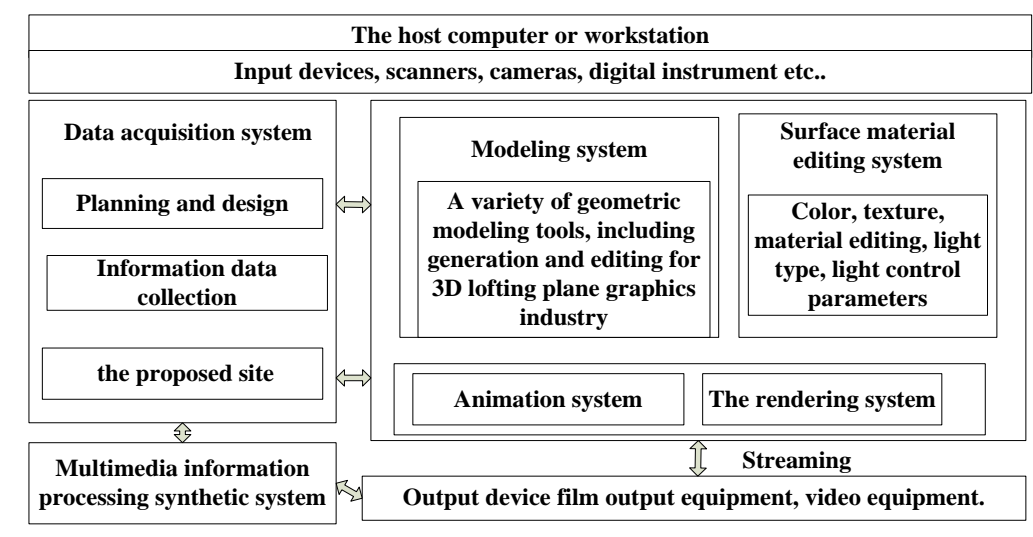

Fig.3 The composition of three-dimensional animation system

The production of three-dimension animation technology involves quite a wide range of knowledge. Three-dimensional animation in early times mainly adopts advanced programming languages. With the rapid development of computer technology and the born and evolution of graphics, the production technology of three-dimensional animation has made a progress from abstract computer programming to interactive generation phase, and forms three-dimensional animation system which gathers production, edit, debugging and rendering into one. Therefore, from the point of producing principle and technology of three-dimensional animation, we can divide three-dimensional technology into five sorts, which are key frame animation, character animation, particle animation, physically- based animation and group animation. Fig.4 displays the process of three-dimensional animation production technology.

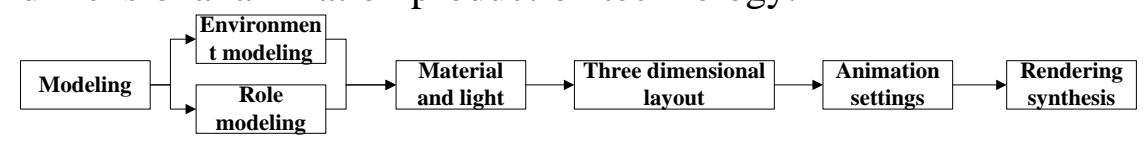

Fig.4 The process of three-dimensional animation production technology

Analysis of three-dimensional animation technology based on scripting language.The basic approach of script animation as follows:

Firstly, in 3ds maxscript, set the key word of animation to be animate. We have to locate the animation expression or corresponding holding function (block-expression) in the brackets behind animate on. Thus, when the animation property of 3ds max object changes, we can create key frame, without considering the real state of animation key frame. If we locate motion expression or function behind animate off, it will prevent the object from creating key frame, when the animation property changes. The syntax format is : [with] animate <boolean_expr $>$ (). No matter it is animate on or animate off, the following expression in the brackets will not influence the state of the animation recorder button. That is to say, even if the animation recorder button is not opened, we can still set up animation motion.

Secondly, corresponding with the time slider in 3ds system, maxscript also use the key word at time. Within the time specified by at time <time>, we can set up any animation properties of the objects.

Thirdly, all animations in 3ds max are controlled by Controller. Animation controller mainly refers to three control items of the object called Position, Rotation and Scale. The animation controller of these three control items is PRS controller. The controller default of Position is Bezier controller, and for Rotation it is TCB controller, while for Scale it is Bezier controller. Users can see these items through Track View and Motion. The items have access to Track View and Motion. Most controllers which can produce key frames will storage animation value into . Keys. The items mainly include controller embedded by 3ds max and all third party plug-in item. The common approach is getPointControllers \{<editable_mesh_node> | <editable_spline_node> \}. The animation parameters which can be visited by controllers mainly include: 
$<$ parameter $>$. Controller---controller of parameter

<parameter $>$. isAnimiated--- indicate whether parameter can be served as Boolean value of animation

<parameter>. Keys---the key frame group to obtain parameter control

Fourthly, key frame function: Key frame function of controller mainly refers to related operation of key frames in animation settings. It includes the addition, deletion, movement and index of key frame. The general format is <key frame function $><$ controller $><$ time $>$ [ \#select ] [ \#interpolate ]

Control of motion trail in three-dimensional animation script.It is very important for threedimension animation design to constrain motion trail of object and control of key frame as well as setting of property of object, which makes the animation of the object in the setting move with corresponding rule and requirement. Taking the motion trail of globule as an example:

$\mathrm{s}=$ sphere pos:[10,10,0] radius:15 ---create globule

$\mathrm{h}=$ helix pos:[-50,0,0] radius1:30 radius2:100 height:90 turn 3 ---create the motion trail of globule.

s.pos.controller = path_constraint () ---allocate motion controller for globule

s.pos.controller.appendtarget h 100 ---add path controller of globule

animate on -- setting animation for globule

( slidertime $=0$ f s.pos.controller.setweight 1100 ) --end script

Firstly, create a globule and a helix in the setting. In order to make the path of globule visible, we set the property of helix path as visibility. Then, attach motion path controller to globule, so that it can move according to the fixed helix path. After the program runs, the effect pictures of Frame 0, Frame 30 and Frame 80 as follows:
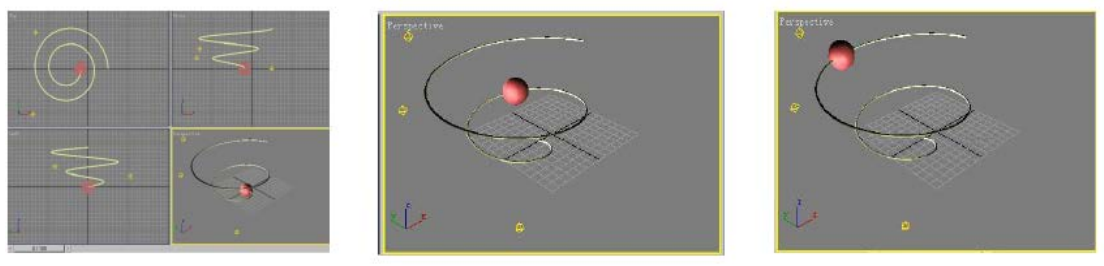

Fig.5 Motion trail under different frames

\section{Analysis of aesthetic degree in three-dimensional animation movies}

The accomplishment of three-dimensional animation requires design of the figures, 3D modelling, texture attachment, binding skeletal animation, output and some other processes. Although the processes seem to be more complex than those of two-dimensional animation industry, that's not the truth. Two-dimensional animation industry needs more people to portray. It also requires very precise professional skills. However, the processes of three-dimensional animation industry seem to be complicated, but it is really convenient to operate. The division of three-dimensional animation software is very clear and elaborate. Even though you don' t have thorough knowledge of arts, you will be adequate to the job through software commands, which can obtain the liberation on the staff. Three-dimensional animation technology can be used for simulating real objects. Due to its characters of authenticity, accuracy and unlimited modifiability, it is widely used in various fields, such as Fig. 6 Toy Story, Fig. 7 Despicable Me, Fig. 8 Avatar and so on. Many excellent threedimensional animation movies strike the animation markets continuously. We can even say that there exists some technical supports of three-dimensional animation in two-dimensional animation. Three-dimension animation successfully capture the heart of the audience through colourful scene as well as exciting visual effect. Of course, it is also an outcome of three-dimensional technology development. With the trend of our times, three-dimensional animation takes place of twodimensional animation and turns to be the master of animation market. 


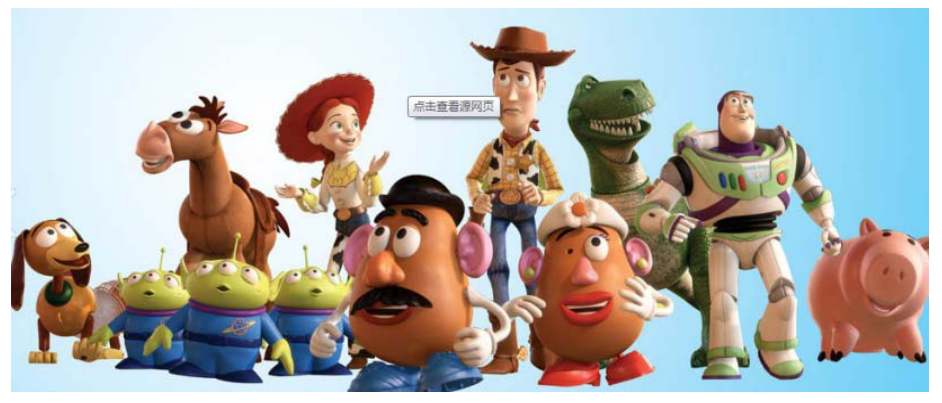

Fig. 6 Toy Story

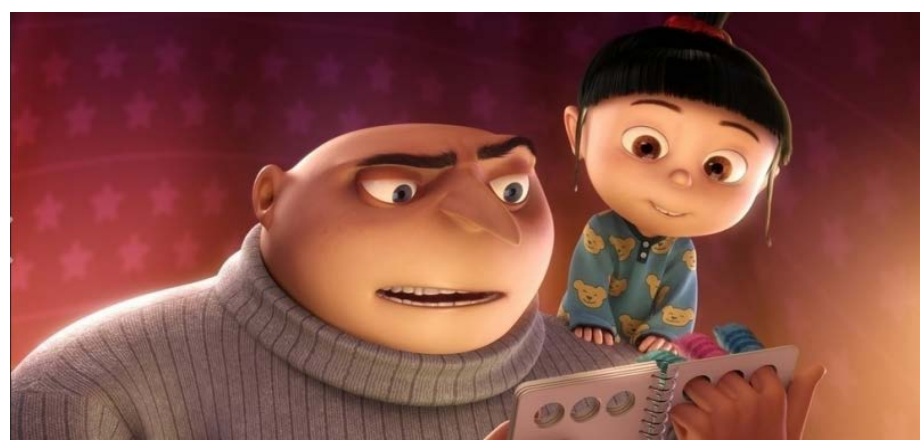

Fig.7 Despicable Me

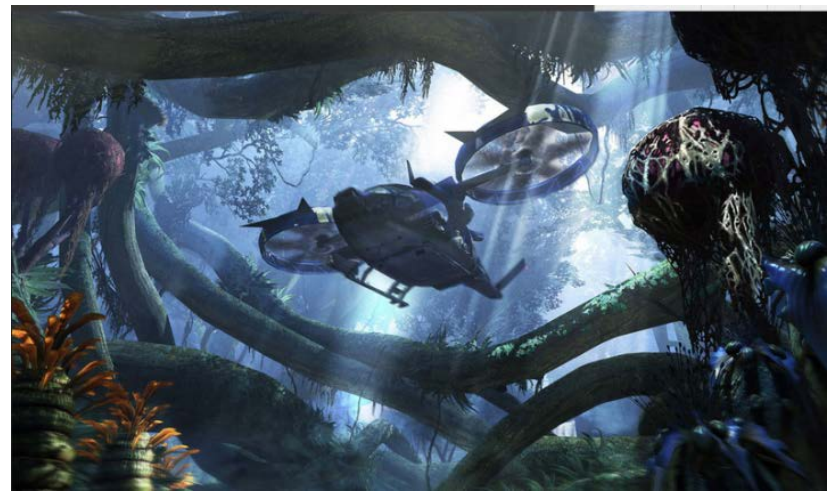

Fig. 8 Avatar

Firstly, create realistic models by simulating real person and physical objects, such as woods, bodies, faces, animals, buildings and so on. To create these models, we need to apply three modelling methods of MAYA software, which are UNRBS, POLYGON and SUBDIVISION. And POLYGON modelling is the main part of application for models in movies and games. POLYGON modelling is also named Polygon modelling, which is different from NURBS and SUBDIVISION. Polygon modelling can split the UV of models, which helps to solve the later problem, like overstretch the maps occurring during the process of mapping. Texture artist will carefully elaborate the UV which is after splitting and offer a real skin to the model, which avoids the problem of stretch or dislocation as much as possible.

Secondly, focus on science fiction movies. Lots of buildings, living beings, plants and even the world that we may have never seen can be virtualized and realized by three-dimensional animation technology. Through the design of original artists, we often see some living beings, plants and the world that seem to be not existing in the real world. For example, the actor rides on the dragon and roams in the sky, which can't be done in the real world. Three-dimensional animation technology can provide virtual scene. For instance, the fierce dragon. We can use video camera to shoot the actor's motion and performance process of riding a dragon, then combine the real shooting with three-dimensional animation by synthetic methods, which makes everything possible. 


\section{Conclusions}

In the movie and television field, the excellent works of three-dimensional animation effect has been emerging in endlessly for the past few years and gains higher and higher technical innovations one step after another. The application of three-dimensional animation effect is very extensive, which attracts more and more animators to throw themselves into it. However, it still can't meet the demands of the market. Three-dimensional animation effect is so charming that plenty of animation masters are all keen on it and create many classic works through three-dimensional animation technology.

The thesis proposes that there exists a more concise method to control the properties of fluid through analyzing and studying Maya Fluid Effects, after taking advantage of maxscript scripting language program to control the motion trails of the objects in three-dimensional animation. Combine particle to work on fluid effect, which can not only make fluid effect construct the sense of reality, but possess the control of particle. Three-dimensional animation effects technology makes continuous innovation in animated movies, whose significance provides theoretical and practical basis for the technology of animation industry in the future.

\section{References}

[1] Yu Lina. The Roles of Computers in Role Making of Three-dimensional Animation Movies [J]. Movies and Television Media,2011(15):178-179.

[2] Li Leiming. Analysis of Personalized Creations of the Roles in Three-dimensional Animation Movies [J]. Film Literature,2010(1).

[3] Bi Luyu. The Expression Styles of Lighting Design in Three-dimensional Aimation[J]. Great Performances,2011(12).

[4] Zeng Yanting, Quan quan. Analysis of Visual Elements in Three-dimensional Aimation[J]. Film Literature,2010(2).

[5] Tan Haichuan. Research of VRay Layer Rendering Technology Based on Three-dimensional Animation [J].Journal of Nanjing Vocational and Technical College,2013,18(6):12-16.

[6] Han Zhijia, Yang Suixian, Wu Yaqun. Research of Realistic Rendering Technology of Products[J].Mechanical Design and Research, 2006, (4):72.

[7] Lei Qing. The Application of Three-dimensional Animation Effects in Film and Television Advertising. [J]. Journal of Academy of Art in Inner Mongolia University, 2008, (4).

[8] Jin Dongxu. Practical Tutorial on Three-dimensional Animation Design[M] . Electronic Industry Press, 2010.

[9] Kang Kai. The Development and Status Analysis of Three-dimensional Animation in China[J].Film Literature, 2008,(17).

[10] Wu Danfang. The Aesthetic Analysis of Three-dimensional Animation Arts[J] . Modern Business Trade Industry, 2008, (1). 